\title{
An Energy Distance Aware Clustering Protocol with Dual Cluster Heads Using Niching Particle Swarm Optimization for Wireless Sensor Networks
}

\author{
Dexin $\mathrm{Ma}^{1}$ and Pengmin $\mathrm{Xu}^{2}$ \\ ${ }^{1}$ Communication College, Qingdao Agricultural University, Qingdao 266109, China \\ ${ }^{2}$ Network Management Center, Qingdao Agricultural University, Qingdao 266109, China \\ Correspondence should be addressed to Pengmin Xu; xupmnc@163.com
}

Received 29 September 2014; Accepted 15 January 2015

Academic Editor: Shengwei Mei

Copyright (C) 2015 D. Ma and P. Xu. This is an open access article distributed under the Creative Commons Attribution License, which permits unrestricted use, distribution, and reproduction in any medium, provided the original work is properly cited.

\begin{abstract}
Energy efficient utilization is an important criteria and factor that affects the design of wireless sensor networks (WSNs). In this literature, we propose an energy distance aware clustering protocol with Dual Cluster Heads using Niching Particle Swarm Optimization (DCH-NPSO). The protocol selects two cluster heads in each cluster, the Master Cluster Head (MCH) and the Slave Cluster Head (SCH), and the selection needs to consider the network state information carefully and deliberately. Simulation results show that the protocol we proposed can balance the energy dissipation and extend the network lifetime effectively.
\end{abstract}

\section{Introduction}

The application of WSNs becomes more wide in recent years; there are a large number of typical applications, such as environment surveillance and exploration, target tracking, and volcano activity monitoring [1]. The energy of wireless sensor nodes is usually supplied by dry/lithium batteries, and, in some dangerous situations, the replacement or recharge of batteries is impossible. So, energy-efficient utilization is an important issue for WSNs, while clustering is as an energyefficient technique and is often used to prolong the sensor network's lifetime [2].

There are several optimization problems in the whole information transmission process, WSN issues, such as clustering, localization, data fusion, and deployment that are often formulated as optimization problems [3]. Traditional mathematical optimization methods need tremendous computation efforts, and with the problem scale increase, the computation grows up exponentially. As to implement on tiny wireless sensor nodes, an optimization method that needs moderate and even minimal computation efforts yet generates better results is needed. Bioinspired optimization methods are computation efficient in contrast to traditional mathematical methods [4]; the authors [5] have demonstrated and compared the general mathematical formulation for this kind of problems. In this paper, we take advantage of bioinspired optimization to resolve clustering formation.

Particle swarm optimization (PSO) is a bioinspired optimization, and it is simple, effective, and popular optimization method [6]. It has been used to address WSN issues such as clustering, deployment, localization, and date aggregation [3]. Niching is also inspired by nature [7]; different species must compete to survive by taking on different roles. Niching is a significant technique for multimodal optimization, and within the framework of PSO, some niching methods have been proposed and developed $[8,9]$.

Several clustering protocols have been proposed for WSNs, LEACH [10] is a famous clustering protocol and selects nodes with a probability to act as cluster heads periodically. BCDCP [11] makes use of the base station to select clusters and routing path and perform randomized shift of cluster heads.

Particle swarm optimization has been used to resolve WSN issues such as clustering, localization, deployment, and data fusion [3]. A protocol using PSO [12] aimed to minimize the intracluster distance and optimize the energy dissipation 
of the network. A clustering particle swarm optimization [13] has been presented for dynamic optimization and employed a hierarchical clustering method to locate multiple optima for dynamic optimization problems.

In this literature, we propose an energy distance aware clustering protocol with Dual Cluster Heads using Niching Particle Swarm Optimization (DCH-NPSO). The protocol generates two cluster heads in each cluster using Niching $\mathrm{PSO}$, a Master Cluster Head (MCH) and a Slave Cluster Head $(\mathrm{SCH})$. The $\mathrm{MCH}$ receives and aggregates the data that have been collected from its member nodes and then sends it to the $\mathrm{SCH}$; the $\mathrm{SCH}$ transmits the aggregated data to the sink directly. In our designed scheme, $\mathrm{MCH}$ does not communicate with the sink directly, that can balance the energy consumption. The simulation results show that the protocol can balance the network loads, minimize the energy consumptions, and prolong the network lifespan of WSNs.

\section{System Model}

2.1. Network Model. We assume a number of $N$ wireless sensor nodes distributed among an $M \times M$ squared field randomly. Sensed data are gathered in a periodical way, and we define each period as a round. Each round contains three phase, a set-up phase uses DCH-NPSO to part the network into clusters and selects $\mathrm{MCH}$ and $\mathrm{SCH}$ using Niching PSO, a steady phase when data collected and aggregated by the $\mathrm{MCH}$, and a finish phase followed when data is transmitted to the BS (Base Station, also called sink node); the steady phase should be longer than other phases to minimize the energy dissipation. The $\mathrm{MCH}$ is used for data collection and data aggregation. The aggregated data are sent to the SCHs, and then the SCHs transmit it to the sink node directly and get some information through a piggyback manner to save more energy. The partial structure of the network in a round is as shown in Figure 1.

We make assumptions about the network model as follows.

(i) All nodes are equipped with small batteries, so each node has initial energy allocated and the energy is constrained.

(ii) A BS situates far away from the sensing field, and it has no energy constraints.

(iii) Each node has its location information. There are a number of existing works on localization in WSNs [14-16]. For example, we use the solution proposed in [16] to obtain the localization.

(iv) All nodes are equipped with power control units to adjust the transmit power according the distance.

(v) All nodes have no mobility.

2.2. Radio Model. The radio model we adopt is as used in [10]. In this model, the transmitter dissipates energy to drive the amplifier and the radio components, and the receiver dissipates energy to drive the radio components. The transmitter has power control ability to properly set the
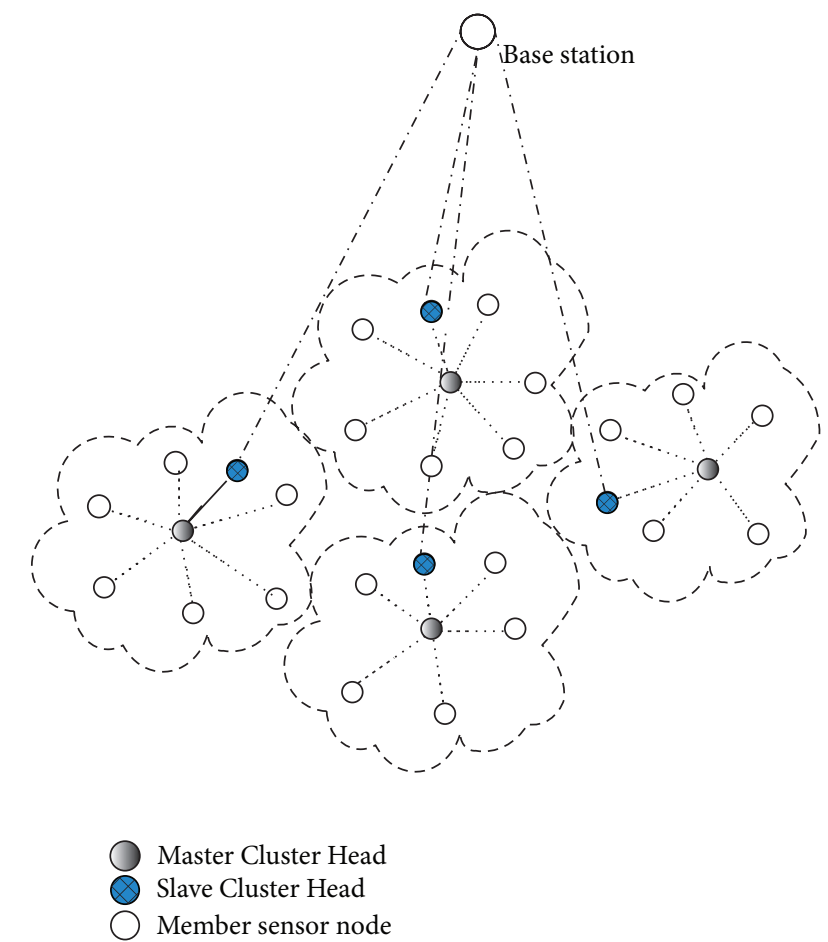

FIGURE 1: DCH-NPSO partial network architecture.

amplifier according to the distance and can be turned off to avoid receiving unintended sensing data, to minimize the energy consumption. The energy dissipation for transmit an $l$-bit packet over distance $d$ is

$$
\begin{aligned}
E_{\mathrm{Tx}}(l, d) & =E_{\mathrm{elec}}(d)+E_{\mathrm{amp}}(l, d) \\
& = \begin{cases}l \times E_{\mathrm{Tx}}+l \times \varepsilon_{f s} \times d^{2}, & d \leq d_{0} \\
l \times E_{\mathrm{Tx}}+l \times \varepsilon_{m p} \times d^{4}, & d>d_{0},\end{cases}
\end{aligned}
$$

where $d_{0}$ is the threshold distance [17] and is given via $d_{0}=\sqrt{\varepsilon_{f_{s}} / \varepsilon_{m p}}$. The energy dissipation that the receiver requires to receive an $l$-bit message is $E_{\mathrm{Rx}}(l)=l \times E_{\text {elec }}$. $E_{\text {elec }}$ is the energy consumed per bit to run the receiver or the transmitter circuit, $\varepsilon_{f s}$ and $\varepsilon_{m p}$ depend on the transmitter amplifier model, the energy for data aggregation $E_{\mathrm{DA}}$ is set to $5 \mathrm{~nJ} / \mathrm{bit} / \mathrm{message}$ [18].

\section{DCH-NPSO Protocol}

Our proposed protocol takes full advantage of Niching PSO for clustering, takes the network states information into account deliberately, and selects $\mathrm{SCH}$ to balance the energy dissipation to improve the energy efficiency.

3.1. Niching Particle Swarm Optimization. Particle swarm optimization is a bionic optimization that is inspired by nature such as bird flocking and fish school, and it was first put forward by Kennedy and Eberhart [19]. In a classic PSO, a number of $m$ particles fly at a certain speed, respectively, in a $D$-dimension space, and each particle has a fitness value on 
the basis of the fitness function which is defined as related to the problems. The particles fly towards the position defined as personal best position and global best position (i.e., the best position refers to a position that gives the best fitness value). During each iteration, the particles' velocity and position are updated based on the following equations, respectively:

$$
\begin{gathered}
v_{i d}^{k+1}=\omega v_{i d}^{k}+c_{1} \xi\left(p_{i d}^{k}-x_{i d}^{k}\right)+c_{2} \eta\left(p_{g d}^{k}-x_{i d}^{k}\right), \\
x_{i d}^{k+1}=x_{i d}^{k}+v_{i d}^{k+1},
\end{gathered}
$$

where $v_{i d}$ is particle $i$ 's velocity in $d$ dimension, $k$ stands for the current iteration, $x_{i d}$ is the particle $i$ 's position in $d$ dimension, $\omega$ is the inertia weight, deciding how the particle inherit present velocity, $c_{1}$ and $c_{2}$ are acceleration coefficient (or called learning factor) which are positive constants normally, $\xi$ and $\eta$ are stochastic numbers between 0 and $1, p_{i d}$ is the particle's best position, and $p_{g d}$ is the global best position [20,21].

When the issue target is to find multiple global optima, niching methods are valuable and efficient. Niching PSO searched in parallel, so the chance of getting trapped into a local optimum is reduced [7,9]. There has been a lot of existing work on niching methods [7-9]; in this paper, we use a ring topology niching method as [7], and each particle interacts with its immediate neighbors only.

3.2. The Fitness Function of NPSO. The fitness function is closely correlated with the features of the problem and determines the property of the optimal solution directly. So, the design of the fitness function is very important. Based on $[3,12]$, we consider the conditions that affect the system performance and define the fitness function deliberately and comprehensively. At first, we should take the residual energy of the sensor node into account, because if the node becomes the $\mathrm{MCH}$, it dissipates more energy than the member nodes. Then, the distance between the MCH and its member nodes should be considered as the $\mathrm{MCH}$ takes charge of gathering data from its member nodes. At last, we should take the distance between the SCH and the BS into account as SCH sends the aggregated data to the BS directly. We define the fitness function $f(i)$ of node $i$ based on these considerations:

$$
\begin{aligned}
f(i) & =\alpha f_{1}(i)+\beta f_{2}(i)+\gamma f_{3}(i), \\
f_{1}(i) & =\frac{\sum_{i=1}^{N} E\left(n_{i}\right)}{\sum_{k=1}^{k_{\mathrm{opt}}} E\left(\mathrm{CH}_{p, k}\right)}, \\
f_{2}(i) & =\frac{\sum_{k=1}^{k_{\mathrm{opt}}} \sum_{\forall n_{i} \in C_{p, k}} d\left(n_{i}, \mathrm{CH}_{p, k}\right)}{\left|C_{p, k}\right|}, \\
f_{3}(i) & =\frac{d\left(\mathrm{CH}_{p, k}, \mathrm{BS}\right)}{k_{\mathrm{opt}}} \sum_{k=1}^{k_{\mathrm{opt}}} d\left(\mathrm{CH}_{p, k}, \mathrm{BS}\right), \\
0 & \leq \alpha, \beta, \gamma \leq 1, \quad \alpha+\beta+\gamma=1,
\end{aligned}
$$

where $n_{i}$ is the sensor node $i, E\left(n_{i}\right)$ is the residual energy of sensor nodes $i, \mathrm{CH}_{p, k}$ is the nodes that belong to cluster $C_{k}$ of particle $p, N$ is the number of node alive in the network, and $k_{\mathrm{opt}}$ is the optimal number of clusters in the network $[10,12]$, so $f_{1}(i)$ takes the network's energy efficiency into account and select the nodes that has more residual energy as cluster head. $\left|C_{p, k}\right|$ is the number of nodes that belong to cluster $C_{k}$ of particle $p, d\left(n_{i}, \mathrm{CH}_{p, k}\right)$ is the Euclidean distance between them, so $f_{2}(i)$ minimizes the intracluster distance between the $\mathrm{CH}$ and its member nodes, and $f_{3}(i)$ minimizes the distance between the $\mathrm{CH}$ s and the BS. The fitness function $f(i)$ has the maximum of the residual energy and the minimum of the distance between the nodes among the cluster and the distance between the $\mathrm{CH}$ and the BS. So, based on the above fitness function, we choose the node that has the maximum fitness value as $\mathrm{MCH}$ and the suboptimal solution as $\mathrm{SCH}$, that is the optimal selections during that round.

3.3. DCH-NPSO Setup. The proposed protocol runs in periodic way, and we use Niching PSO and the fitness function (4) to select the optimal set of CHs and their member nodes. The sensed data transmissions begin after the clusters have been selected; each $\mathrm{MCH}$ gathers and aggregates the data collected from its members. The aggregated data are sent to the $\mathrm{SCH}$, and the SCH transmits the data to the BS directly. The flowchart of DCH-NPSO is shown in Figure 2.

The MCH sets up a TDMA schedule for its member nodes to avoid collection during data transmission, allowing the radio units to be turned off at all times except the transmission period for each member sensor. And we use a fixed spreading code and CSMA (Carrier Sense Multiple Access), a similar approach used in [22] during data transmission to minimize and balance the energy dissipation. Once the $\mathrm{MCH}$ finishes gathering data from its member sensors, the $\mathrm{MCH}$ performs data aggregation and sends the aggregated data to the $\mathrm{SCH}$ and then the $\mathrm{SCH}$ sends the aggregated data to the BS directly.

\section{Simulation}

We perform the simulations to evaluate the proposed protocol's performance. We assume that in a $100 \mathrm{~m} \times 100 \mathrm{~m}$-squared sensing area, 100 sensor nodes are distributed randomly, and the $\mathrm{BS}$ is situated at $(50,175) \mathrm{m}$, the data message length is 75 bytes with 15 -byte packet header. The simulation parameters are given in Table 1, in which the radio parameters are as described in Section 2 and particle swarm optimization is as described in Section 3, and we use ring topology as in [7] to form niching. The performances of our proposed protocol are compared with clustering protocol LEACH, LEACH-C [10], and PSO-C [12].

We use the number of rounds to measure the number of nodes alive during the simulation; it as shown in Table 2. We can see that, as for LEACH, the first node is "dead" (i.e., the node has consumed all its initial energy) in 725 rounds, $\mathrm{LEACH}-\mathrm{C}$ in 843 rounds and PSO-C in 954 rounds, while DCH-PSO in 1134 rounds, and our proposed protocol prolongs the network lifetime about 410 rounds compared to $\mathrm{LEACH}$. The reason is that LEACH and LEACH-C do not 


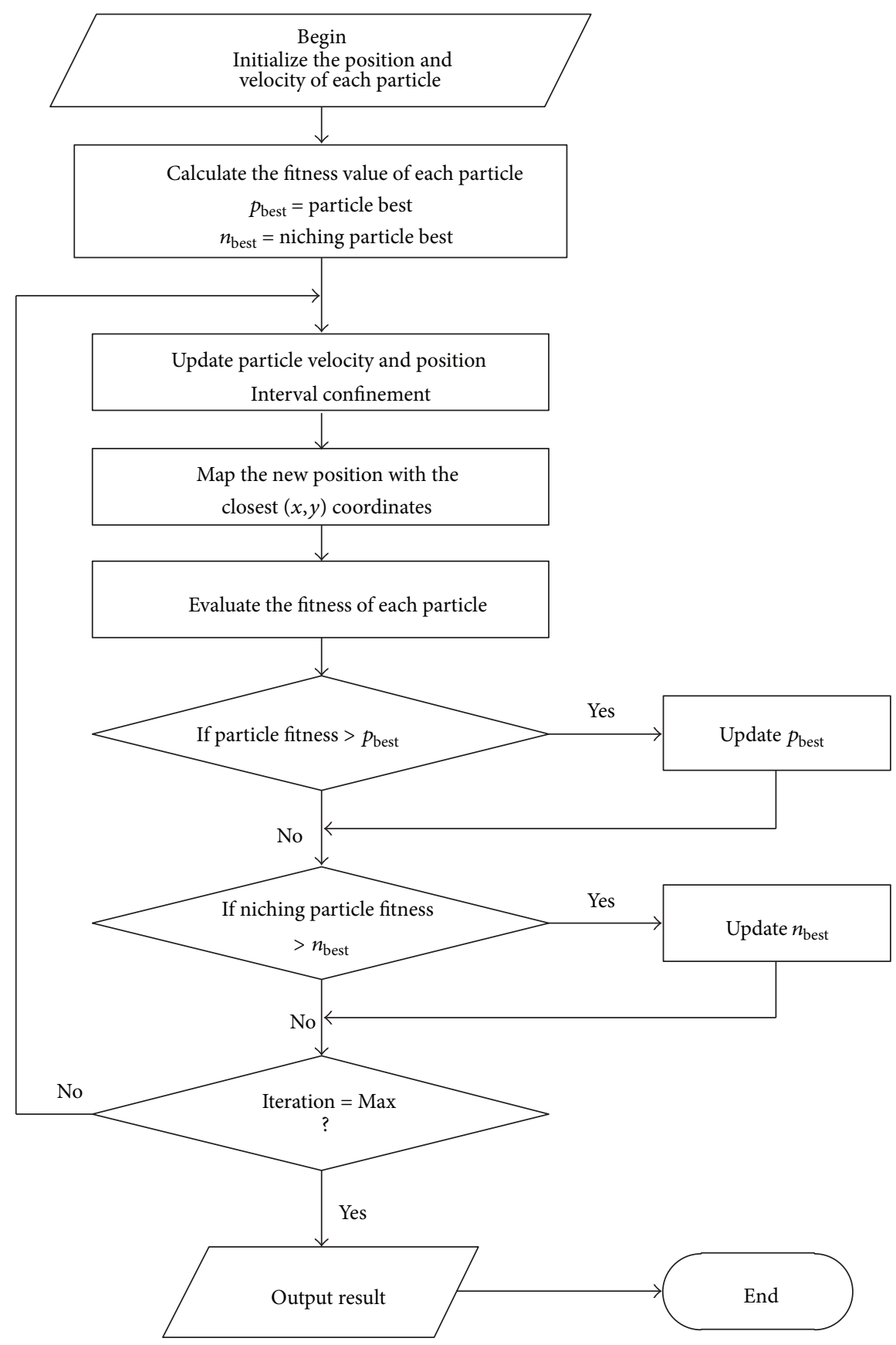

FIGURE 2: The flowchart of the DCH-NPSO protocol for cluster setup.

take the residual energy of sensor nodes into account and select cluster head randomly, although PSO-C can prolong the network lifetime compared to LEACH and LEACH-C; it still does not balance the energy consumption. Our protocol DCH-NPSO takes the residual energy into account and selects $\mathrm{SCH}$ in each cluster, which balances and minimizes the energy consumption.

Figure 3 shows the total data messages received at the BS, the plot clearly indicates the effectiveness of the proposed protocol that delivers more data messages than LEACH, $\mathrm{LEACH}-\mathrm{C}$, and PSO-C, because the proposed protocol takes advantage of higher energy sensors such as MCHs by considering the residual energy of the nodes and selects $\mathrm{SCH}$ to balance the energy dissipation. Thus, more data messages are transmitted to the BS. In contrast, LEACH does not consider the residual energy of a sensor node when choosing the $\mathrm{MCH}$ and may choose the $\mathrm{MCH}$ with insufficient energy to stay alive during the data transmission phase. Although PSO-C takes the node's residual energy and the intracluster distance into consideration, it does not take actions to balance the energy consumption, while our protocol divides the network into clusters evenly, takes the nodes residual energy and the 
TABLE 1: Simulation parameters for DCH-NPSO.

\begin{tabular}{lc}
\hline Parameter & Value \\
\hline$E_{\text {elec }}$ & $50 \mathrm{~nJ} / \mathrm{bit}$ \\
$\varepsilon_{f s}$ & $10 \mathrm{pJ} / \mathrm{bit} / \mathrm{m}^{2}$ \\
$\varepsilon_{m p}$ & $0.0013 \mathrm{pJ} / \mathrm{bit}^{4} \mathrm{~m}^{4}$ \\
$E_{\mathrm{DA}}$ & $5 \mathrm{~nJ} / \mathrm{bit} / \mathrm{message}$ \\
$c_{1}$ & $2.5 \sim 0.5$ \\
$c_{2}$ & $0.5 \sim 2.5$ \\
$\omega$ & $0.8 \sim 0.3$ \\
\hline
\end{tabular}

TABLE 2: Network lifespan comparison.

\begin{tabular}{lccc}
\hline Protocol & First node dead & Half nodes dead & All nodes dead \\
\hline LEACH & 725 & 1053 & 1418 \\
LEACH-C & 843 & 1136 & 1580 \\
PSO-C & 954 & 1308 & 1636 \\
DCH-NPSO & 1134 & 1545 & 1788 \\
\hline
\end{tabular}

intracluster distance into account during the $\mathrm{MCH}$ selection, and selects $\mathrm{SCH}$ to balance the energy dissipation.

As can be seen from Figure 3 and Table 2, the protocol we proposed can prolong the network lifetime and transmit more sensed data to the BS and thus improves the system robustness and enhances the system scalability. LEACH, LEACH-C, and PSO-C, on the other hand, either generate an uneven distribution of cluster heads throughout the network or do not balance the energy dissipation. Our protocol produces performs even clustering distribution and selects $\mathrm{SCH}$ to balance the energy consumption.

\section{Conclusion}

In this literature, we have presented an energy distance aware clustering protocol with Dual Cluster Heads using Niching Particle Swarm Optimization (DCH-NPSO). We uses Niching PSO to select $\mathrm{MCH}$ and $\mathrm{SCH}$ in a cluster, taking the factors such as residual energy and the distance into account to balance and minimize the energy consumption. Results from the simulations show that the proposed protocol using Niching PSO prolongs the network lifetime and delivers more data to the BS compared to LEACH, LEACH-C, and PSOC. Our future work includes the multihop routing among the network and explores rational parameters of Niching PSO to further improve the energy efficiency and the system performance.

\section{Conflict of Interests}

The authors declare that there is no conflict of interests regarding the publication of this paper.

\section{Acknowledgments}

The authors would like to thank the editors and the anonymous reviewers for their constructive and valuable comments, which are helpful for improving the quality of this

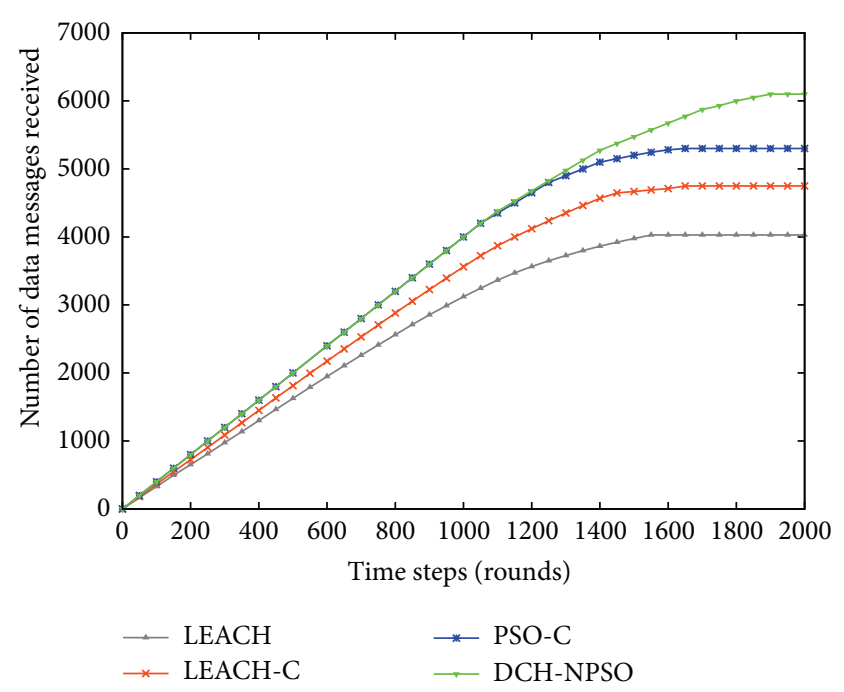

FIGURE 3: The flowchart of the DCH-NPSO protocol for cluster setup.

paper. This work was supported by Qingdao Science and Technology Plan under Grants no. 14-2-3-56-nsh and no. 14-8-3-6-nsh and Shandong Province Independent Innovation Achievements of Major Projects under Grant no. 2011ZHZX1A0406.

\section{References}

[1] J. Yick, B. Mukherjee, and D. Ghosal, "Wireless sensor network survey," Computer Networks, vol. 52, no. 12, pp. 2292-2330, 2008.

[2] A. A. Abbasi and M. Younis, "A survey on clustering algorithms for wireless sensor networks," Computer Communications, vol. 30, no. 14-15, pp. 2826-2841, 2007.

[3] R. V. Kulkarni and G. K. Venayagamoorthy, "Particle swarm optimization in wireless-sensor networks: a brief survey," IEEE Transactions on Systems, Man and Cybernetics Part C: Applications and Reviews, vol. 41, no. 2, pp. 262-267, 2011.

[4] R. Xu, J. Xu, and D. C. Wunsch, "A comparison study of validity indices on swarm-intelligence-based clustering," IEEE Transactions on Systems, Man, and Cybernetics, Part B: Cybernetics, vol. 42, no. 4, pp. 1243-1256, 2012.

[5] N. M. Abdul Latiff, C. C. Tsimenidis, and B. S. Sharif, "Performance comparison of optimization algorithms for clustering in wireless sensor networks," in Proceedings of the IEEE International Conference on Mobile Adhoc and Sensor Systems (MASS '07), pp. 1-4, October 2007.

[6] Y. del Valle, G. K. Venayagamoorthy, S. Mohagheghi, J.-C. Hernandez, and R. G. Harley, "Particle swarm optimization: basic concepts, variants and applications in power systems," IEEE Transactions on Evolutionary Computation, vol. 12, no. 2, pp. 171-195, 2008.

[7] X. Li, "Niching without niching parameters: particle swarm optimization using a ring topology," IEEE Transactions on Evolutionary Computation, vol. 14, no. 1, pp. 150-169, 2010.

[8] A. E. R. Brits and F. van den Bergh, "A niching particle swarm optimizer," in Proceedings of the Asia-Pacific Conference on Simulated Evolution and Learning (SEAL '02), pp. 692-696, 2002. 
[9] A. P. Engelbrecht, B. S. Masiye, and G. Pampard, "Niching ability of basic particle swarm optimization algorithms," in Proceedings of the IEEE Swarm Intelligence Symposium (SIS '05), pp. 397400, June 2005.

[10] W. B. Heinzelman, A. P. Chandrakasan, and H. Balakrishnan, "An application-specific protocol architecture for wireless microsensor networks," IEEE Transactions on Wireless Communications, vol. 1, no. 4, pp. 660-670, 2002.

[11] S. D. Muruganathan, D. C. F. Ma, R. I. Bhasin, and A. O. Fapojuwo, "A centralized energy-efficient routing protocol for wireless sensor networks," IEEE Communications Magazine, vol. 43, no. 3, pp. S8-S13, 2005.

[12] N. M. A. Latiff, C. C. Tsimenidis, and B. S. Sharif, "Energyaware clustering for wireless sensor networks using particle swarm optimization," in Proceedings of the 18th Annual IEEE International Symposium on Personal, Indoor and Mobile Radio Communications (PIMRC '07), pp. 1-5, September 2007.

[13] S. X. Yang and C. H. Li, "A clustering particle swarm optimizer for locating and tracking multiple optima in dynamic environments," IEEE Transactions on Evolutionary Computation, vol. 14, no. 6, pp. 959-974, 2010.

[14] D. Niculescu and B. Nath, "Ad hoc positioning system (APS) using AOA," in Proceedings of the IEEE 22nd Annual Joint Conference of the IEEE Computer and Communications Societies (INFOCOM '03), vol. 3, pp. 1734-1743, San Francisco, Calif, USA, March 2003.

[15] T. He, C. Huang, B. M. Blum, J. A. Stankovic, and T. Abdelzaher, "Range-freelocalization schemes for large scale sensor networks," in Proceedings of the 9th Annual International Conference on Mobile Computing and Networking (MobiCom '03), pp. 81-95, September 2003.

[16] P. Kumar, A. Chaturvedi, and M. Kulkarni, "Geographical location based hierarchical routing strategy for wireless sensor networks," in Proceedings of the International Conference on Devices, Circuits and Systems (ICDCS '12), pp. 9-14, Coimbatore, India, March 2012.

[17] T. Rappaport, Wireless Communications: Principles \& Practice, Prentice-Hall, Upper Saddle River, NJ, USA, 1996.

[18] A. Wang, W. R. Heinzelman, and A. P. Chandrakasan, "Energyscalable protocols for battery-operated microsensor networks," in Proceedings of the IEEE Workshop on Signal Processing Systems (SiPS '99), pp. 483-492, Taipei, Taiwan, October 1999.

[19] J. Kennedy and R. Eberhart, "Particle swarm optimization," in Proceedings of the IEEE International Conference on Neural Networks, pp. 1942-1948, December 1995.

[20] Y. Shi and R. Eberhart, "A modified particle swarm optimizer," in Proceedings of the IEEE International Conference on Evolutionary Computation, pp. 69-73, IEEE, Anchorage, Alaska, USA, May 1998.

[21] T. Blackwell, "A study of collapse in bare bones particle swarm optimization," IEEE Transactions on Evolutionary Computation, vol. 16, no. 3, pp. 354-372, 2012.

[22] K. Pahlavan and A. Levesque, Wireless Information Networks, John Wiley \& Sons, New York, NY, USA, 1995. 

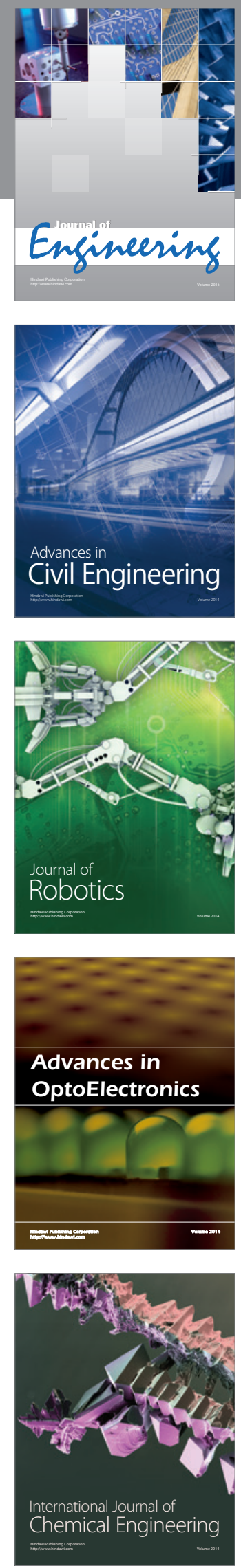

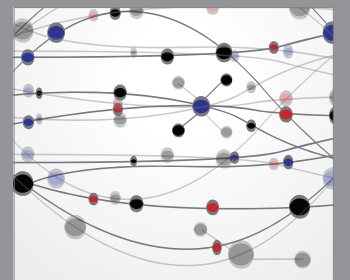

The Scientific World Journal
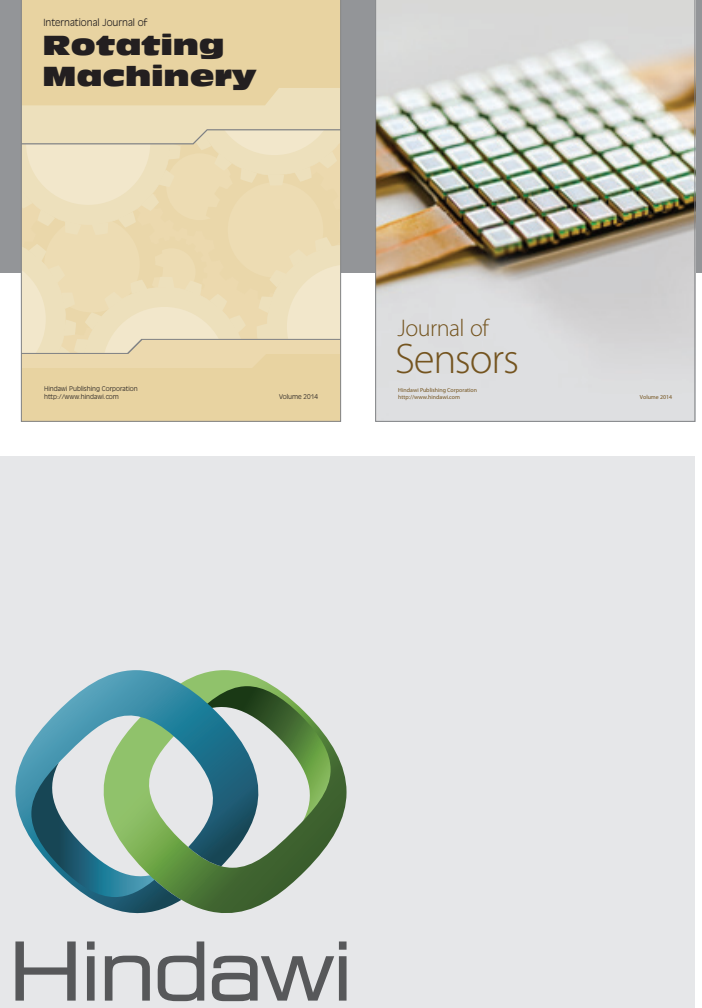

Submit your manuscripts at http://www.hindawi.com
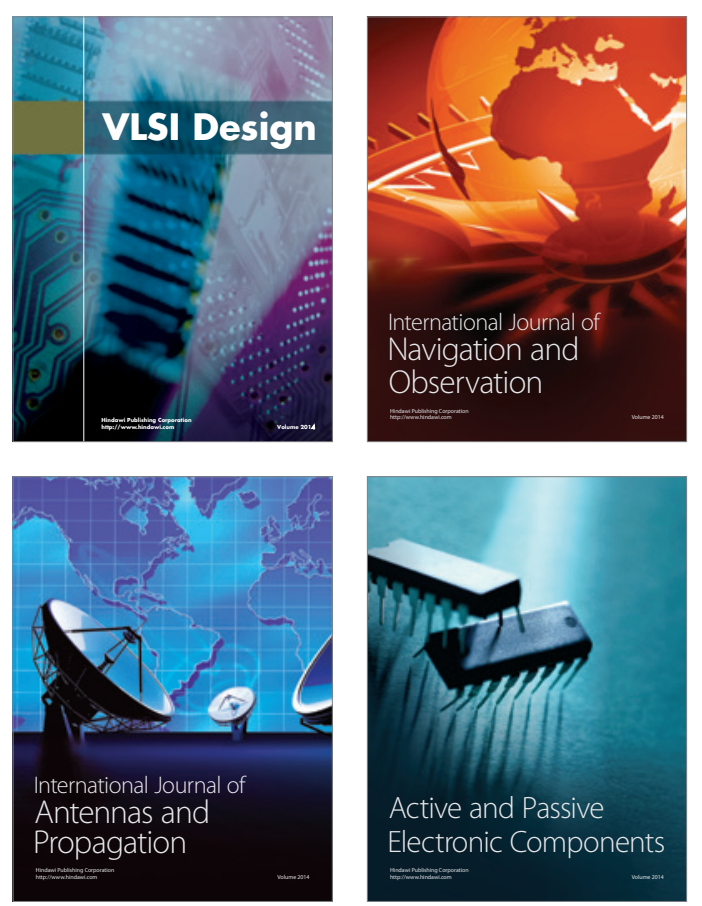
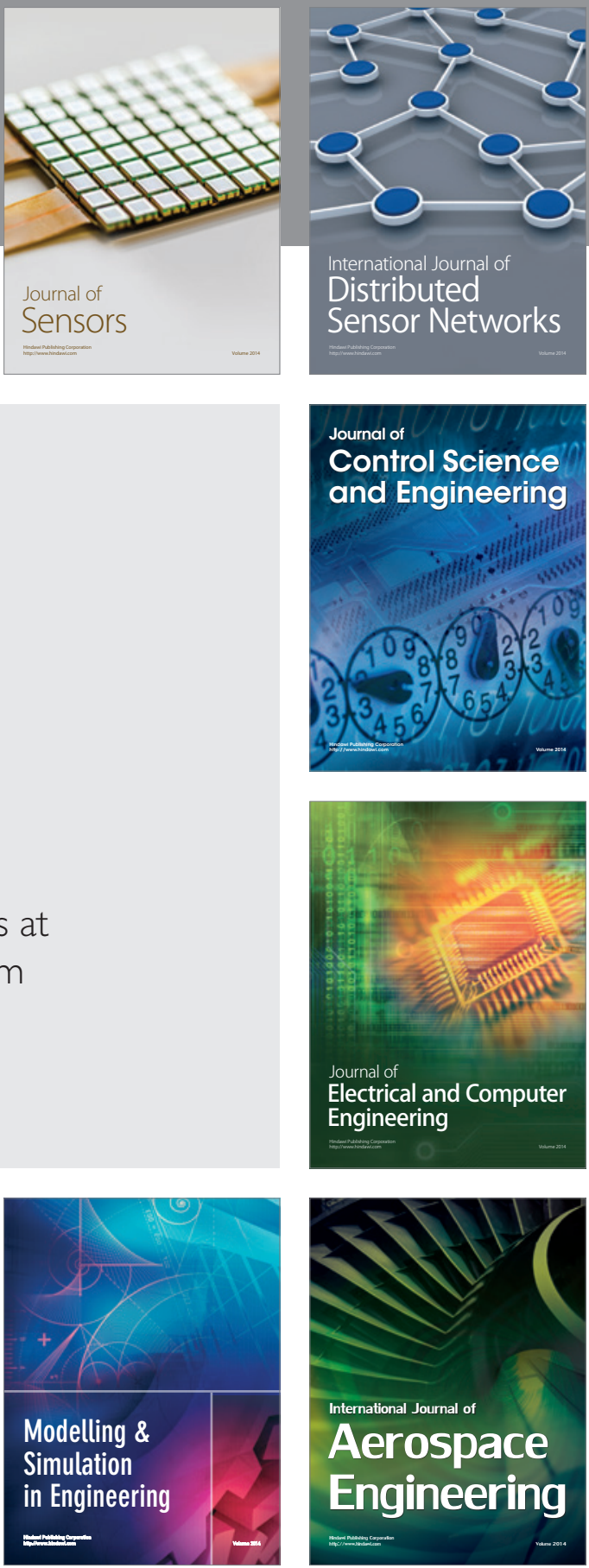

Journal of

Control Science

and Engineering
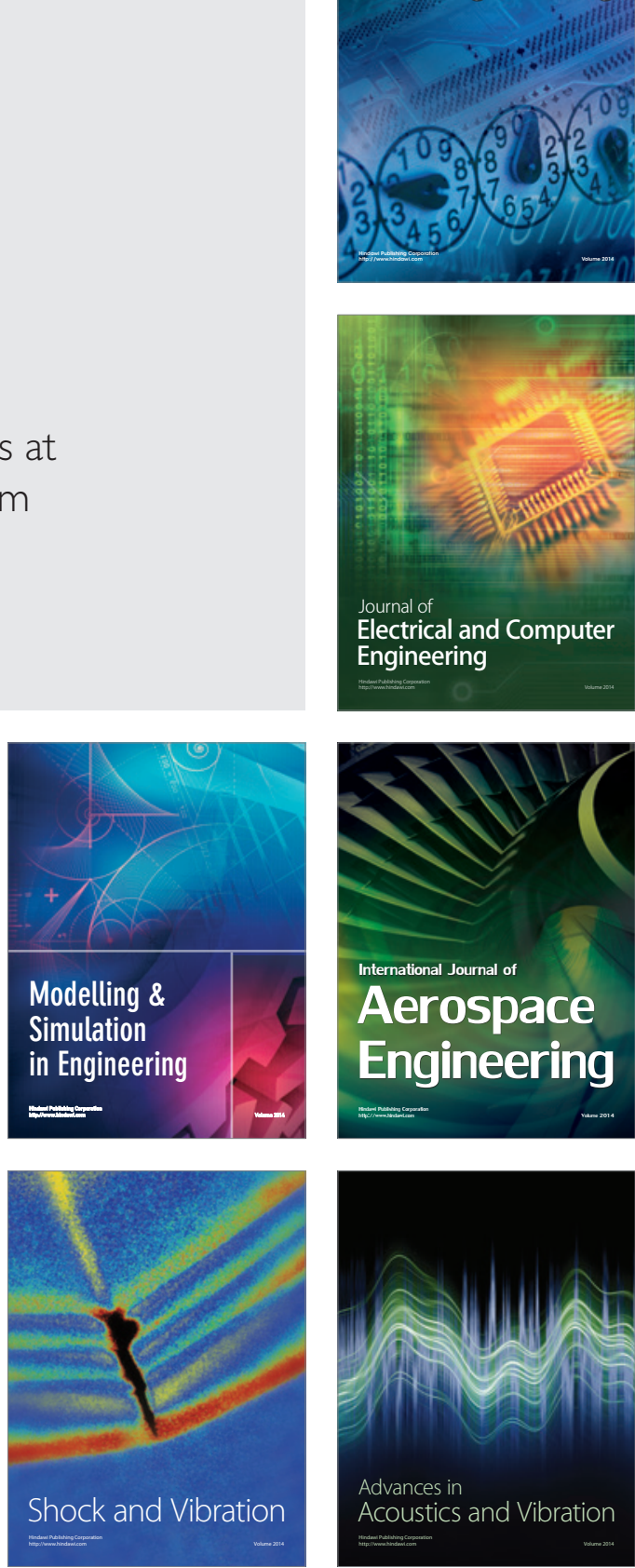